\title{
Nonlinear spin control by terahertz-driven anisotropy fields
}

\author{
S. Baierl'1, M. Hohenleutner', T. Kampfrath ${ }^{2}$, A. K. Zvezdin ${ }^{3,4,5}$, A. V. Kimel ${ }^{4,6}$, R. Huber ${ }^{1 \star}$ \\ and R. V. Mikhaylovskiy ${ }^{6 \star}$
}

Future information technologies, such as ultrafast data recording, quantum computation or spintronics, call for ever faster spin control by light ${ }^{1-16}$. Intense terahertz pulses can couple to spins on the intrinsic energy scale of magnetic excitations ${ }^{5,11}$. Here, we explore a novel electric dipole-mediated mechanism of nonlinear terahertz-spin coupling that is much stronger than linear Zeeman coupling to the terahertz magnetic field5,10. Using the prototypical antiferromagnet thulium orthoferrite $\left(\mathrm{TmFeO}_{3}\right)$, we demonstrate that resonant terahertz pumping of electronic orbital transitions modifies the magnetic anisotropy for ordered $\mathrm{Fe}^{3+}$ spins and triggers large-amplitude coherent spin oscillations. This mechanism is inherently nonlinear, it can be tailored by spectral shaping of the terahertz waveforms and its efficiency outperforms the Zeeman torque by an order of magnitude. Because orbital states govern the magnetic anisotropy in all transition-metal oxides, the demonstrated control scheme is expected to be applicable to many magnetic materials.

Ultrafast magnetization control has become a key goal of modern photonics, with a broad variety of successful concepts emerging at a fast pace. Examples include light-induced spin reorientation in canted antiferromagnets ${ }^{3}$, vectorial control of magnetization by light ${ }^{6}$, photoinduced antiferromagnet-ferromagnet phase transitions, optical modification of the exchange energy ${ }^{4,14}$ and driving spin precessions via nonlinear magneto-phononic coupling ${ }^{7,16}$. Despite this remarkable progress, most of the photon energy in all known concepts using visible and near-infrared light is inactive with respect to the light-spin interaction, and avoiding dissipation of large excess energies requires special care.

In contrast, intense electromagnetic pulses at terahertz frequencies may interface spin dynamics directly on their intrinsic energy scales $^{5,11}$. The magnetic field component of few-cycle terahertz pulses has been used to coherently control magnons in the electronic ground state by direct Zeeman interaction ${ }^{5,11}$. Because magnetic dipole coupling is typically weak, however, terahertz-driven spin excitation has been confined to the linear response regime. Massive nonlinearities, such as terahertz-induced phase transitions ${ }^{17,18}$ and terahertz lightwave electronics ${ }^{19-22}$, in turn, have been realized by all-electric coupling to the charge degree of freedom. Apart from pioneering work on electromagnons in multiferroic $\mathrm{TbMnO}_{3}$ (ref. 13), efficient ways to exploit the terahertz electric field for the control of magnetic order have been missing.

Here, we introduce a conceptually new universal route to control magnetism with terahertz electric fields. The strength and direction of the magnetic anisotropy in practically all materials is determined by the coupling of electronic orbital states to ordered spins.
Therefore, an ultrashort electric field pulse changing the orbital state of electrons abruptly can lead to a sudden modification of the magnetic anisotropy. In our proof-of-concept experiment, we exploit intense, phase-locked terahertz pulses to achieve such an abrupt change of the magnetic anisotropy, which in turn triggers magnon oscillations with large amplitudes that scale quadratically with the terahertz field strength.

Non-thermal pumping of orbital transitions in the optical range is known to induce a nonlinear spin-charge coupling on ultrashort timescales ${ }^{23,24}$. In the terahertz spectral range, this concept can be applied to any material in which selected low-energy electronic transitions change the magnetic anisotropy, for example in oxides containing both $3 d$ and $4 f$ ions (for example, orthoferrites, manganites, garnets and ferroborates) and in $3 d$-compounds such as hematite $\alpha-\mathrm{Fe}_{2} \mathrm{O}_{3}$. However, despite the anticipated strong impact of the preparation of non-thermal orbital states on the anisotropy field, terahertz spin control exploiting orbital transitions has remained largely unexplored ${ }^{25,26}$.

Figure 1 illustrates the fundamental idea of our experiment for the case of the prototypical antiferromagnet $\mathrm{TmFeO}_{3}$. This material crystallizes in a distorted perovskite structure (Fig. 1a). The four iron spins (blue arrows) per unit cell occupy two antiferromagnetically coupled sublattices, whose spin orientations are mutually canted by the Dzyaloshinskii-Moriya interaction ${ }^{27}$. The ${ }^{3} \mathrm{H}_{6}$ ground state of the paramagnetic rare-earth $\mathrm{Tm}^{3+}$ ions is fully split by the crystal field into a series of singlets with a characteristic energy spacing of $\sim 1-10 \mathrm{meV}$ (ref. 28). The angular momenta of these states are coupled with the $\mathrm{Fe}^{3+}$ spins by exchange and dipolar interactions, which set the magnetic anisotropy. Thermal population of the singlet states within the ${ }^{3} \mathrm{H}_{6}$ multiplet changes the magnetic anisotropy as a function of temperature $(T)$, leading to spin reorientation phase transitions ${ }^{29}$. In the $\Gamma_{2}$ phase $\left(T<T_{1}=80 \mathrm{~K}\right)$, the antiferromagnetic vector $\mathbf{G}$ is aligned along the crystallographic $z$ axis, whereas it lies along the $x$ axis in the $\Gamma_{4}$ phase $\left(T>T_{2}=90 \mathrm{~K}\right)$. For $T_{1}<T<T_{2}$ ( $\Gamma_{24}$ phase), $\mathbf{G}$ rotates continuously in the $x-z$ plane (Fig. 1b, equation (6) in Methods, Supplementary Fig. 1 and Supplementary Movie 1). The spin dynamics support two eigenmodes, the quasi-ferromagnetic (q-FM) and the quasi-antiferromagnetic ( $\mathrm{q}-\mathrm{AFM}$ ) one. Instead of thermal activation, resonant pumping of electronic transitions between orbital states of the rare-earth ions by terahertz pulses may be expected to abruptly modify the magnetic anisotropy to trigger coherent magnon oscillations (Fig. 1c and Supplementary Movie 2).

We excited a $60-\mu \mathrm{m}$-thick window of $\mathrm{TmFeO}_{3}$ with intense few-cycle terahertz transients generated by tilted-pulse-front optical rectification

\footnotetext{
'Department of Physics, University of Regensburg, Regensburg 93053, Germany. ${ }^{2}$ Department of Physical Chemistry, Fritz Haber Institute of the Max Planck Society, Berlin 14195, Germany. ${ }^{3}$ Prokhorov General Physics Institute, Russian Academy of Sciences, Moscow 119991, Russia. ${ }^{4}$ Moscow Technological University (MIREA), Moscow 119454, Russia. ${ }^{5}$ Moscow Institute of Physics and Technology (State University), Dolgoprudny 141700, Russia.

${ }^{6}$ Radboud University, Institute for Molecules and Materials, Nijmegen 6525 AJ, The Netherlands. *e-mail: rupert.huber@physik.uni-regensburg.de; r.mikhaylovskiy@science.ru.nl
} 


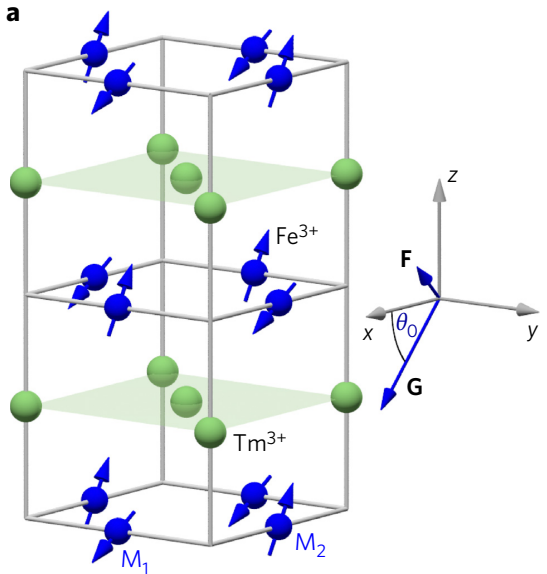

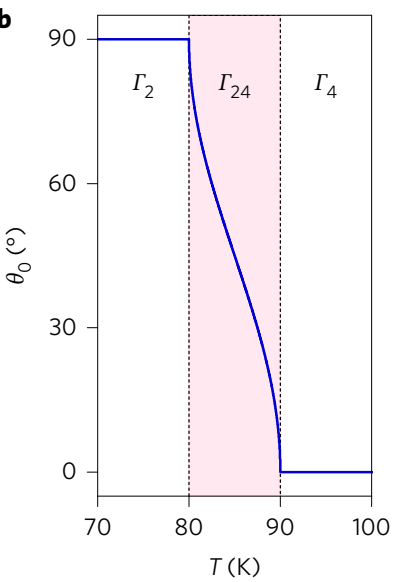
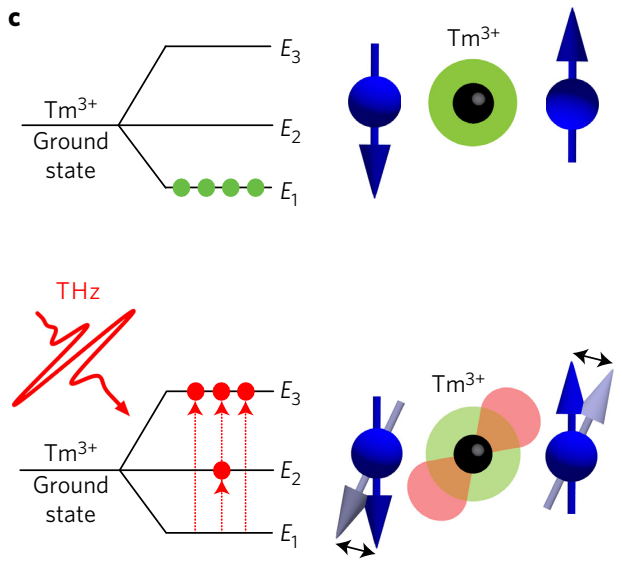

Figure 1 | Principle of spin control by a terahertz-induced anisotropy torque. a, Spin and lattice structure of $\mathrm{TmFeO}_{3}$ shown in the $\Gamma_{24}$ phase. Green/blue spheres: $\mathrm{Tm}^{3+} / \mathrm{Fe}^{3+}$ ions. Oxygen atoms are not shown, for clarity. The iron spins (blue arrows) form two antiferromagnetically coupled sublattices $\mathbf{M}_{1}$ and $\mathbf{M}_{2}$, which are mutually canted by the Dzyaloshinskii-Moriya interaction, giving rise to a weak ferromagnetic moment $\mathbf{F}=\mathbf{M}_{1}+\mathbf{M}_{2}$. In the $\Gamma_{24}$ phase (that is, for $80 \mathrm{~K}<T<90 \mathrm{~K}$ ), the antiferromagnetic vector $\mathbf{G}=\mathbf{M}_{1}-\mathbf{M}_{2}$ encloses a finite angle $0^{\circ}<\theta_{0}<90^{\circ}$ with the $x$ axis. $\mathbf{b}$, Spin reorientation phase transitions. In the $\Gamma_{2}$ phase $(T<80 \mathrm{~K})$, the antiferromagnetic vector $\mathbf{G}$ is aligned along the crystallographic $z$ axis, whereas it lies along the $x$ axis above $T=90 \mathrm{~K}\left(\Gamma_{4}\right.$ phase). In the $\Gamma_{24}$ phase, $\mathbf{G}$ rotates continuously in the $x-z$ plane. $\mathbf{c}$, The crystal field splits the ground state ${ }^{3} \mathrm{H}_{6}$ of the rare-earth Tm ${ }^{3+}$ ions into several energy levels with an energy spacing of $\sim 1-10 \mathrm{meV}$ (schematic level scheme). The corresponding orbital wavefunctions set the magnetic anisotropy for the iron spins in thermal equilibrium (upper panel). Ultrafast transitions between these energy levels resonantly induced by terahertz pulses should exert an abrupt torque on the spins and act as an efficient trigger for coherent spin dynamics (lower panel). The small canting angle is not shown, for clarity.
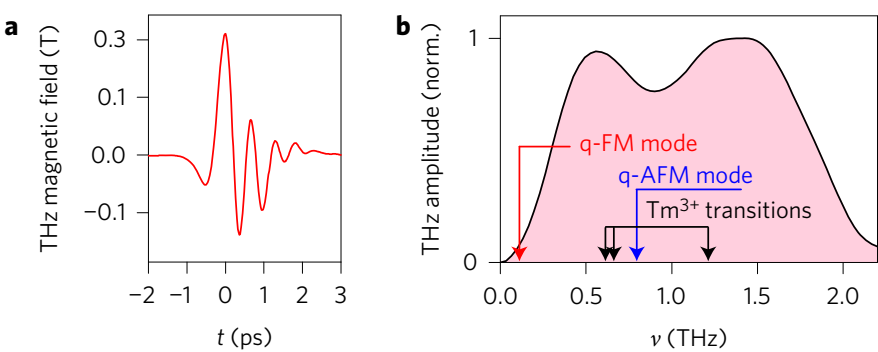

C

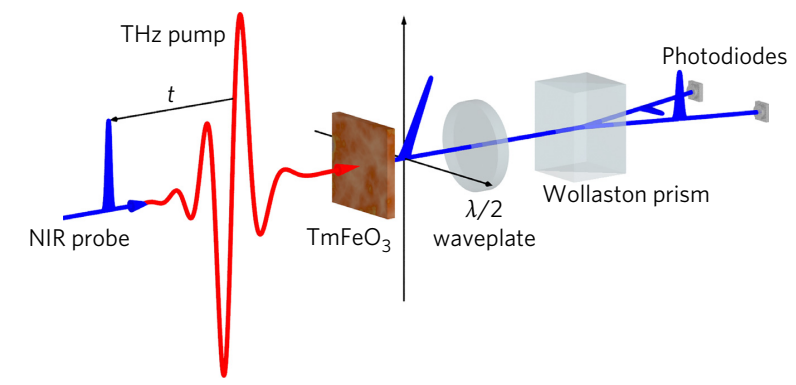

d

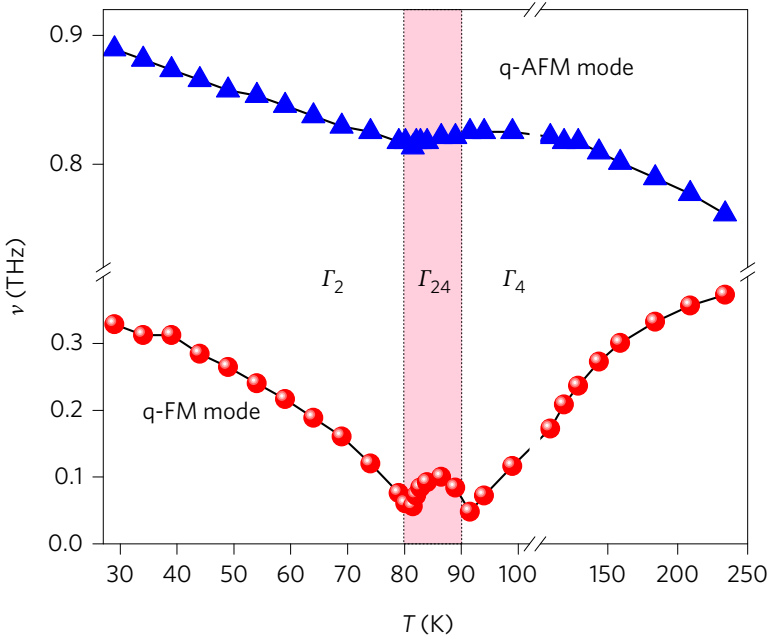

Figure 2 | Overview of the experiment. $\mathbf{a}$, Electro-optically detected terahertz transients used to excite magnon/Tm ${ }^{3+}$ resonances in $\mathrm{TmFeO}_{3}$. $\mathbf{b}$, Amplitude spectrum of the waveform shown in $\mathbf{a}$. Arrows indicate the frequencies of the magnon/Tm ${ }^{3+}$ resonances. $\mathbf{c}$, Schematic of the experiment. The terahertz pump (red) and near-infrared probe pulses (NIR, blue) are collinearly focused onto the $\mathrm{TmFeO}_{3}$ sample with variable delay time $t$. Using a $\lambda / 2$ plate, a Wollaston prism and two balanced photodiodes, terahertz-induced magnetic dynamics in $\mathrm{TmFeO}_{3}$ are measured by polarization rotation of the probe pulses. $\mathbf{d}$, Resonance frequencies of the q-FM (red circles) and q-AFM (blue triangles) modes in dependence on sample temperature T. Black curves are guides to the eye.

of near-infrared laser pulses ${ }^{11}$. The terahertz peak field can be tuned up to $B_{\mathrm{THz}}=0.3 \mathrm{~T}$ without changing the waveform (Fig. 2a). The spectral content lies between 0.1 and $2 \mathrm{THz}$, covering both magnon modes of $\mathrm{Fe}^{3+}$ spins and several transitions of the $\mathrm{Tm}^{3+}$ ground state multiplet ${ }^{28}$ (Fig. 2b). Excitation of phonons can be excluded as they feature frequencies above $3 \mathrm{THz}$ (refs 28,30). The induced ultrafast magnon dynamics is revealed by tracking the polarization rotation imprinted by the Faraday effect and magnetic linear dichroism on co-propagating near-infrared $30 \mathrm{fs}$ probe pulses (Fig. 2c, Supplementary Figs 2 and 3). Both the q-FM and q-AFM modes are excited (Supplementary Fig. 4) and their frequencies exhibit characteristic temperature dependences (Fig. 2d). Close to the phase transitions, we observe a dramatic softening of the q-FM mode down to a frequency $(v)$ of $50 \mathrm{GHz}$, in agreement with theory ${ }^{28}$.

Next, we systematically vary $B_{\mathrm{THz}}$ while keeping the sample in the $\Gamma_{24}$ transition phase $(T=84.5 \mathrm{~K})$, where pumping of the rare-earth states should have maximum impact on the magnetic anisotropy. Figure 3a shows the dynamic polarization rotation as a function of the delay time $t$ between the terahertz pump and the optical probe. Each transient is normalized by the corresponding terahertz peak field (Supplementary Fig. 5 gives a quantitative analysis of the magnetization deflection angles). The signal exhibits an 



Figure 3 | Nonlinear terahertz-magnon interaction. a, Normalized magnon traces for various terahertz excitation fields $B_{\mathrm{THz}}$. Whereas quasimonochromatic oscillations are found for the lowest terahertz field, a low-frequency oscillation is superimposed onto the dynamics for higher pump fields. $\mathbf{b}$, Amplitude spectra of the time-domain data shown in a allow the identification of q-FM and q-AFM modes at 100 and $830 \mathrm{GHz}$, respectively. $\mathbf{c}$, Scaling of the amplitudes from $\mathbf{b}$. The q-AFM mode (blue triangles) scales linearly with the terahertz field strength, whereas the q-FM mode (red circles) shows a nonlinear dependence on it. Error bars denote the standard deviation interval for the terahertz amplitude, arising from uncertainties in the terahertz spot size and the repeatability of the polarizer angle. d, Deviation of the experimental field-scaling of the q-FM mode with terahertz field strength from a linear behaviour (red data points) and terahertz-induced anisotropy torque exerted on the spins (black curve) as computed by our model for various temperatures at the magnetic phase transitions. The nonlinear behaviour vanishes outside the $\Gamma_{24}$ phase. Error bars take account of the uncertainty in the extracted nonlinearity owing to noise in the measurements.

oscillatory behaviour with two quasi-monochromatic components at frequencies of 0.1 and $0.8 \mathrm{THz}$ corresponding to the q-FM and q-AFM modes, respectively (Fig. 3b). Most remarkably, the relative strength of the q-FM mode grows with increasing terahertz peak fields. Figure $3 c$ summarizes the amplitude of both modes as a function of $B_{\mathrm{THz}}$. The q-AFM mode scales linearly with the terahertz driving field, as expected for the linear Zeeman interaction. In contrast, the q-FM mode shows a distinctly nonlinear increase, which is well fit by a superposition of linear and quadratic functions of the peak field. The nonlinearity vanishes when the crystal leaves the $\Gamma_{24}$ phase (Fig. 3d).

One can show (section 'Model for purely magnetic interaction' in the Methods and Supplementary Fig. 6) that the Zeeman torque exerted by the terahertz magnetic field on the $\mathrm{Fe}^{3+}$ spins cannot explain the nonlinear excitation of the q-FM mode. In contrast, the terahertz electric field can influence the magnetic system: the point group of the orthoferrites allows for an anisotropic energy term that scales quadratically with the electric field. Due to this term, the terahertz driving field may change the magnetic anisotropy (section 'Role of the terahertz electric field' in the Methods). Importantly, the link between the terahertz electric field and the spins is not restricted to a certain microscopic mechanism. In our

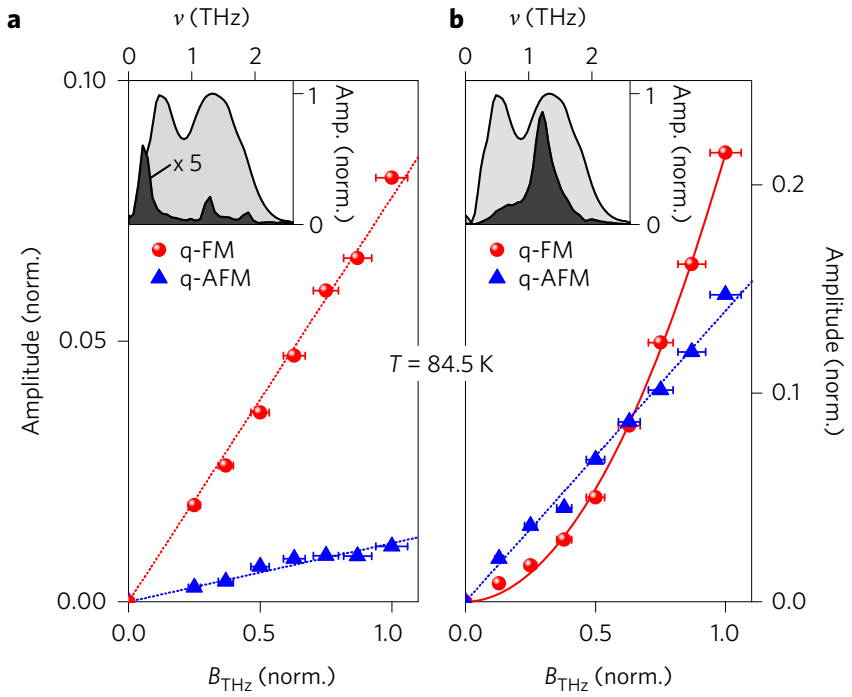

Figure 4 | Control of terahertz-induced nonlinear torque by spectral shaping. a, Low-pass filtering the pristine terahertz spectrum (grey shaded curve in inset) results in the black shaded spectrum (inset), featuring a dominant maximum at the q-FM resonance frequency. Main graph: linear scaling of the $\mathrm{q}-\mathrm{FM}$ amplitude obtained by Zeeman excitation with the constrained terahertz spectrum (red circles). The q-AFM mode (blue triangles) is suppressed because the terahertz amplitude at the corresponding frequencies is strongly reduced by the low-pass filter. $\mathbf{b}$, The Zeeman-type excitation of the $\mathrm{q}-\mathrm{FM}$ mode is switched off by bandpass filtering (centre frequency, $1.2 \mathrm{THz}$ ) of the terahertz pulse (black/grey: filtered/pristine spectrum). Main graph: $\mathrm{Tm}^{3+}$ excitation triggers a q-FM oscillation, whose amplitude scales quadratically with the terahertz field (red circles). Additionally, a q-AFM magnon (amplitude: blue triangles) is excited by the Zeeman interaction. All curves are normalized to the maximum amplitude of the q-FM mode shown in Fig. 3c. Dotted/solid lines are linear/quadratic fits. Error bars are obtained as in Fig. 3c.

specific experiment, the terahertz electric field resonantly excites electronic transitions between the singlet states of the ${ }^{3} H_{6}$ multiplet of the $\mathrm{Tm}^{3+}$ ions (ref. 28 and Supplementary Fig. 7). The concomitant non-thermal occupation change alters the magnetic anisotropy.

The fact that the nonlinear excitation is achieved most efficiently in the vicinity of the magnetic phase transition temperatures, where the static magnetic anisotropy is effectively zero, confirms that the excitation of the q-FM magnon is caused by the transient anisotropy. Indeed, our model shows that anisotropy changes generated by the electric field of the terahertz pulse can drive large-angle excitations of the magnetic lattice, which behaves 'soft' at these temperatures (section 'Role of the terahertz electric field' in Methods). Figure $3 \mathrm{~d}$ depicts the temperature dependence of the modelled anisotropy torque (black curve) together with the deviation of the q-FM amplitude from a linear scaling with the terahertz field (red data points). Our theory traces the experimental data very well.

To put our interpretation to an ultimate test, we repeated the experiments with spectrally filtered terahertz pulses that selectively excite either the q-FM mode or the electronic transitions in the $\mathrm{Tm}^{3+}$ ions. Indeed, we found a linear dependence of the magnon amplitude on the terahertz field if the q-FM mode was excited selectively (Fig. 4a). A complementary terahertz spectrum (inset of Fig. 4b) that cannot couple by Zeeman interaction, resonantly prepares non-thermal orbital states of the $\mathrm{Tm}^{3+}$ ions, leading to a quadratic scaling (Fig. 4b). These results prove that the nonlinear spin excitation is mediated by the rare-earth ions, which exert a strong effective torque on the $\mathrm{Fe}^{3+}$ spin system. From our measurements, we find that the strength of the anisotropy-mediated spin excitation is eight times larger than the Zeeman interaction for 
terahertz pulses featuring peak magnetic fields of $0.3 \mathrm{~T}$ (see Methods). With these terahertz amplitudes, peak-to-peak magnetization deflection angles of $2.6^{\circ}$ are reached (Supplementary Fig. 5). Neglecting saturation effects, we estimate that upscaling the terahertz electric field to $\sim 3 \mathrm{MV} \mathrm{cm}^{-1}$ (magnetic field: $1 \mathrm{~T}$ ) may suffice for non-thermal switching of the magnetization direction by $90^{\circ}$ via the nonlinear anisotropy torque (Supplementary Fig. 8). Finally, we note that a similar nonlinear scaling of the magnon amplitude with the terahertz field occurs in dysprosium orthoferrite $\left(\mathrm{DyFeO}_{3}\right)$ (Supplementary Fig. 9), another magnetic reference system, underlining the broad applicability of the new concept of terahertz-induced anisotropy torque.

In conclusion, we have demonstrated a novel interface between terahertz fields and the spin system of an antiferromagnet that exploits electric-dipole transitions coupled to the magnetic degree of freedom of electrons. In this way, we have realized the first nonlinear excitation of the amplitude of spin oscillations using terahertz pulses. The spectral sensitivity of the effect and its high efficiency compared to Zeeman excitation open an unprecedented route to further raise the amplitude of terahertz-driven spin deflection using pulse-shaping and coherent control. Throughout the broad class of rare-earth-transition metal compounds, predicted field thresholds ${ }^{8}$ for terahertz-induced magnetic switching may be reduced by an order of magnitude. Our work exploits a new, general concept of electric field control of magnetic excitations by creating hidden states of matter that involve the spin degree of freedom. In the same spirit, one may now investigate the role of other low-energy elementary excitations, such as excitons or phonons ${ }^{16}$, which could change the orbital wavefunctions of nearby atoms and lead to the creation of magnons by a related mechanism. Finally, the new principle of a symmetry-breaking preparation of low-energy non-thermal states may open unforeseen applications in future spin-based devices.

\section{Methods}

Methods and any associated references are available in the online version of the paper.

Received 24 March 2016; accepted 12 August 2016; published online 3 October 2016

\section{References}

1. Beaurepaire, E., Merle, J.-C., Daunois, A. \& Bigot, J.-Y. Ultrafast spin dynamics in ferromagnetic nickel. Phys. Rev. Lett. 76, 4250-4253 (1996).

2. Van Kampen, M. et al. All-optical probe of coherent spin waves. Phys. Rev. Lett. 88, 227201 (2002).

3. Kimel, A. V., Kirilyuk, A., Tsvetkov, A., Pisarev, R. V. \& Rasing, Th. Laser-induced ultrafast spin reorientation in the antiferromagnet $\mathrm{TmFeO}_{3}$. Nature 429, 850-853 (2004).

4. Wall, S., Prabhakaran, D., Boothroyd, A. T. \& Cavalleri, A. Ultrafast coupling between light, coherent lattice vibrations, and the magnetic structure of semicovalent $\mathrm{LaMnO}_{3}$. Phys. Rev. Lett. 103, 097402 (2009).

5. Kampfrath, T. et al. Coherent terahertz control of antiferromagnetic spin waves. Nat. Photon. 5, 31-34 (2011).

6. Kanda, N. et al. The vectorial control of magnetization by light. Nat. Commun. 2, 362 (2011)

7. Kim, K. W. et al. Ultrafast transient generation of spin-density-wave order in the normal state of $\mathrm{BaFe}_{2} \mathrm{As}_{2}$ driven by coherent lattice vibrations. Nat. Mater. 11, 497-501 (2012).

8. Wienhold, S., Hinzke, D. \& Nowak, U. THz switching of antiferromagnets and ferrimagnets. Phys. Rev. Lett. 108, 247207 (2012).

9. Li, T. et al. Femtosecond switching of magnetism via strongly correlated spin-charge quantum excitations. Nature 496, 69-73 (2013).

10. Vicario, C. et al. Off-resonant magnetization dynamics phase-locked to an intense phase-stable terahertz transient. Nat. Photon. 7, 720-723 (2013).

11. Kampfrath, T., Tanaka, K. \& Nelson, K. A. Resonant and nonresonant control over matter and light by intense terahertz transients. Nat. Photon. 7, 680-690 (2013).
12. Matsunaga, R. et al. Light-induced collective pseudospin precession resonating with Higgs mode in a superconductor. Science 345, 1145-1149 (2014).

13. Kubacka, T. et al. Large-amplitude spin dynamics driven by a $\mathrm{THz}$ pulse in resonance with an electromagnon. Science 343, 1333-1336 (2014).

14. Mikhaylovskiy, R. V. et al. Ultrafast optical modification of exchange interactions in iron oxides. Nat. Commun. 6, 8190 (2015).

15. Satoh, T., Iida, R., Higuchi, T., Fiebig, M. \& Shimura, T. Writing and reading of an arbitrary optical polarization state in an antiferromagnet. Nat. Photon. 9, 25-29 (2015).

16. Nova, T. F. et al. An effective magnetic field from optically driven phonons. Nat. Phys. http://dx.doi.org/10.1038/nphys3925 (in the press); ibid. Condens. Mater. Preprint at http://arXiv.org/abs/1512.06351 (2015).

17. Fausti, D. et al. Light induced superconductivity in a stripe-ordered cuprate. Science 331, 189-191 (2011).

18. Liu, M. et al. Terahertz-field-induced insulator-to-metal transition in vanadium dioxide metamaterial. Nature 487, 345-348 (2012).

19. Zaks, B., Liu, R. B. \& Sherwin, M. S. Experimental observation of electron-hole recollisions. Nature 483, 580-583 (2013).

20. Cocker, T. L. et al. An ultrafast terahertz scanning tunnelling microscope. Nat. Photon. 7, 620-625 (2013).

21. Hohenleutner, M. et al. Real-time observation of interfering crystal electrons in high-harmonic generation. Nature 523, 572-575 (2015).

22. Maag, T. et al. Coherent cyclotron motion beyond Kohn's theorem. Nat. Phys. 12, 119-123 (2016).

23. Dodge, J. S. et al. Time-resolved optical observation of spin-wave dynamics. Phys. Rev. Lett. 83, 4650-4653 (1999).

24. Lingos, P. C., Wang, J. \& Perakis, I. E. Manipulating femtosecond spin-orbit torques with laser pulse sequences to control magnetic memory states and ringing. Phys. Rev. B 91, 195203 (2015).

25. Bossini, D. et al. Time-resolved nonlinear infrared spectroscopy of samarium ions in $\mathrm{SmFeO}_{3}$. Phys. Rev. B 87, 085101 (2013).

26. Reid, A. H. M., Rasing, Th., Pisarev, R. V., Dürr, H. A. \& Hoffmann, M. C. Terahertz-driven magnetism dynamics in the orthoferrite $\mathrm{DyFeO}_{3}$. Appl. Phys. Lett. 106, 082403 (2015).

27. White, R. L. Review of recent work on the magnetic and spectroscopic properties of the rare-earth orthoferrites. J. Appl. Phys. 40, 1061-1069 (1969).

28. Srinivasan, G. \& Slavin, A. N. High Frequency Processes in Magnetic Materials Ch. 2 (World Scientific, 1995).

29. Belov, K. P., Volkov, R. A., Goranskii, B. P., Kadomtseva, A. M. \& Uskov, V. V. Nature of the transitions during the spontaneous reorientation of spins in rareearth orthoferrites. Fiz. Tverd. Tela 11, 1148-1151 (1969), Sov. Phys. Solid State 11, 935-938 (1969).

30. Smith, B. T., Yamamoto, J. \& Bell, E. E. Far-infrared transmittance of Tb, Ho, Tm, Er, and Yb orthoferrite. J. Opt. Soc. Am. 65, 605-607 (1975).

\section{Acknowledgements}

The authors thank M. Furthmeier for technical assistance, R.V. Pisarev and A.M. Balbashov for providing samples, T.L. Cocker for discussions and Th. Rasing for continuous support. S.B., M.H. and R.H. were supported by the European Research Council through ERC grant no. 305003 (QUANTUMsubCYCLE) and the Deutsche Forschungsgemeinschaft (DFG) through Collaborative Research Centre SFB 689. A.V.K., R.V.M. and A.K.Z. were supported by the European Community Seventh Framework Programme FP7-NMP-2011-SMALL281043 (FEMTOSPIN), the European Research Council ERC (grant agreement no. 257280, Femtomagnetism), the Foundation for Fundamental Research on Matter (FOM) as well as the Netherlands Organization for Scientific Research (NWO) and the programme 'Leading Scientist' of the Russian Ministry of Education and Science (14.z50.31.0034).

T.K. acknowledges the Deutsche Forschungsgemeinschaft and ERC for support through priority programme SPP 1538 and the ERC grant no. 681917 (TERAMAG), respectively.

\section{Author contributions}

S.B., A.V.K., R.H. and R.V.M. conceived the study, carried out the experiments and analysed the data. A.K.Z. and R.V.M. developed the theoretical model. S.B., M.H., A.V.K., R.H. and R.V.M. wrote the manuscript with feedback from T.K. and A.K.Z. All authors discussed the results.

\section{Additional information}

Supplementary information is available in the online version of the paper. Reprints and permissions information is available online at www.nature.com/reprints. Correspondence and requests for materials should be addressed to R.H. and R.V.M.

\section{Competing financial interests}

The authors declare no competing financial interests. 


\section{Methods}

Sample. A single crystal of $\mathrm{TmFeO}_{3}$ (grown by a floating zone melting technique), with a thickness of $\sim 60 \mu \mathrm{m}$ and lateral dimensions of $\sim 5 \mathrm{~mm}$, was used in the experiments. The plate was cut perpendicularly to one of the crystal's optical axes, which lays in the $y-z$ plane at an angle of $51^{\circ}$ with respect to the $z$ axis. This orientation allows for terahertz excitation of both magnon modes at all temperatures. A constant magnetic field of $0.1 \mathrm{~T}$ saturated the magnetization of the sample.

Set-up. The detailed experimental set-up is presented in Supplementary Fig. 10. A low-noise Ti:sapphire laser amplifier (centre wavelength, $800 \mathrm{~nm}$; pulse energy, $5.5 \mathrm{~mJ}$; repetition rate, $3 \mathrm{kHz}$; pulse duration, $30 \mathrm{fs}$ ) generates intense few-cycle terahertz fields by tilted-pulse-front optical rectification in a cryogenically cooled $\mathrm{LiNbO}_{3}$ crystal. A pair of wire-grid polarizers and different terahertz spectral filters (for their transmission characteristics see insets to Fig. 4) allows for adjustment of the peak fields and the spectral shape of the terahertz pulses. A small portion of the laser pulses (pulse duration, $30 \mathrm{fs}$; pulse energy, $\sim 10 \mathrm{~nJ}$ ) is sent through a mechanical delay line and used as a polarization probe. The terahertz and probe pulses are collinearly focused onto the $\mathrm{TmFeO}_{3}$ sample, which is mounted in a helium cryostat for temperature control. The terahertz-induced rotation of the linear polarization of the probe pulses is analysed by polarization optics consisting of a half-wave plate, a Wollaston polarizer and a pair of balanced silicon photodiodes. A lock-in amplifier is used to record the diode signals as a function of the time delay between the terahertz and the probe pulses.

\section{Theoretical formalism}

Model for purely magnetic terahertz-spin interaction. We describe the dynamics of the quasi-ferromagnetic mode of the weak ferromagnet $\mathrm{TmFeO}_{3}$ using the Lagrangian $L$ and Rayleigh $R$ functions of the angle $\theta$ of the normalized antiferromagnetic vector $\mathbf{G}$ with respect to the crystal axis $x$ (Fig. 1a) and its time derivative $\dot{\theta}$ in the form ${ }^{31,32}$

$$
\begin{gathered}
L=\frac{M_{\mathrm{Fe}}}{2 \gamma^{2} H_{\mathrm{E}}} \dot{\theta}^{2}-\frac{M_{\mathrm{Fe}}}{\gamma H_{\mathrm{E}}} B_{y} \dot{\theta}-W(\theta) \\
R=\frac{\alpha M_{\mathrm{Fe}}}{2 \gamma} \dot{\theta}^{2}
\end{gathered}
$$

Here, $M_{\mathrm{Fe}}$ is the magnetization of a single $\mathrm{Fe}^{3+}$ sublattice, $H_{\mathrm{E}}$ is the effective field of the $d-d$ exchange, $\alpha$ is the Gilbert damping parameter and $W(\theta)$ is the free energy. For $\mathrm{TmFeO}_{3}$ subjected to the magnetic field $\mathbf{B}$ of the terahertz pulse, one has

$$
W(\theta)=K_{1} \sin ^{2} \theta+K_{2} \sin ^{4} \theta-\frac{H_{\mathrm{D}}}{H_{\mathrm{E}}} M_{\mathrm{Fe}}\left(B_{z} \cos \theta-B_{x} \sin \theta\right)
$$

where $H_{\mathrm{D}}$ is the Dzyaloshinskii field, $K_{2}$ is a constant parameter and $K_{1}=2 K_{2}\left(T-T_{2}\right) /\left(T_{1}-T_{2}\right)$ with $T_{1} \approx 80 \mathrm{~K}$ and $T_{2} \approx 90 \mathrm{~K}$. Equation (3) account for the small spin canting angle $\varepsilon=H_{\mathrm{D}} / H_{\mathrm{E}}$, which is a result of the DzyaloshinskiiMoriya interaction. In equation (3), the term containing the magnetic field arises from Zeeman coupling. The equation of motion is

$$
\frac{\mathrm{d}}{\mathrm{d} t}\left(\frac{\partial L}{\partial \dot{\theta}}\right)-\frac{\partial L}{\partial \theta}+\frac{\partial R}{\partial \dot{\theta}}=0
$$

which, in the case of functions (1-3), can be written as

$$
\ddot{\theta}+\omega_{\mathrm{E}} \alpha \dot{\theta}+\omega_{\mathrm{E}} \omega_{\mathrm{A}} w(\theta, T)=\gamma \dot{B}_{y}-\frac{H_{\mathrm{D}}}{H_{\mathrm{E}}} \omega_{\mathrm{E}} \gamma B_{\mathrm{z}} \sin \theta-\frac{H_{\mathrm{D}}}{H_{\mathrm{E}}} \omega_{\mathrm{E}} \gamma B_{x} \cos \theta
$$

where $\gamma$ is the gyromagnetic ratio, $\omega_{\mathrm{E}}=\gamma H_{\mathrm{E}}, \omega_{\mathrm{A}}=\gamma K_{2} / M_{\mathrm{Fe}}$ and $w(\theta, T)=\sin \theta \cos \theta$ $\left(\left(T-T_{2}\right) /\left(T_{1}-T_{2}\right)+\sin ^{2} \theta\right)$. Equation (5) has the form of a generalized sine-Gordon equation and is nonlinear with respect to $\theta$. Assuming $\ddot{\theta}=\theta=0$ in equation (5) one can find the equilibrium orientation $\theta_{0}$ of the antiferromagnetic vector for a given temperature as

$$
\theta_{0}=\left\{\begin{array}{l}
\frac{\pi}{2}, T<T_{1} \\
\arcsin \left(\frac{T-T_{2}}{T_{1}-T_{2}}\right)^{\frac{1}{2}}, T_{1}<T<T_{2} \\
0, T>T_{2}
\end{array}\right.
$$

The temperature dependence $\theta_{0}(T)$ is shown in Fig. $1 \mathrm{~b}$ and Supplementary Fig. 1. We numerically find the solution of equation (5) taking the time trace of the magnetic field $B(t)$ from the experiment (Fig. 2a) and assuming standard initial conditions $\theta(t=0)=\theta_{0}, \dot{\theta}(t=0)=0$. For calculations we take $M_{\mathrm{Fe}}=1,000$ e.m.u. $\mathrm{cm}^{-3}$, $H_{\mathrm{E}}=2 \times 10^{7} \mathrm{Oe}, H_{\mathrm{D}}=2 \times 10^{5}$ Oe and $\omega_{\mathrm{E}} \alpha \approx 0.05 \mathrm{ps}^{-1}$ (ref. 28). The resulting time evolution of angle $\theta(t)$ is shown in Supplementary Fig. 6 for different peak amplitudes of the driving magnetic field $B_{\mathrm{THz}}$ polarized along the $x$ axis, as in the experiment. Although we confirmed the possibility of excitation of the quasi-ferromagnetic mode via the Zeeman mechanism, we did not find any deviation from the linear relation between the maximum amplitude of $\theta$ and $B_{\mathrm{THz}}$ below $0.3 \mathrm{~T}$ for any temperature and orientation of the field in the $x-z$ plane. Our analysis shows that the Zeeman interaction of the $\mathrm{Fe}^{3+}$ sublattices with the terahertz magnetic field cannot lead to the nonlinear excitation observed in the experiment.

Role of the terahertz electric field. To account for the observed nonlinear interaction between the $\mathrm{Fe}^{3+}$ spins and the terahertz pulses we write the free energy term quadratic with respect to the terahertz electric field $\mathbf{E}$ and the antiferromagnetic vector $\mathbf{G}$ as

$$
W_{\text {int }}=\sum_{i, k, l, m} g_{i k l m} G_{l} G_{m} E_{i} E_{k}
$$

which is allowed in centrosymmetric $\mathrm{TmFeO}_{3}$. Here, $g_{i k l m}$ are the components of the nonlinear magnetoelectric susceptibility tensor $\hat{\mathbf{g}}$. The symmetry point group $D_{2 h}^{16}$ of $\mathrm{TmFeO}_{3}$ dictates the form of the tensor $\hat{\mathbf{g}}$, which reads (in the Voigt notation)

$$
\hat{\mathrm{g}}=\left(\begin{array}{cccccc}
g_{11} & g_{12} & g_{13} & 0 & 0 & 0 \\
g_{12} & g_{22} & g_{23} & 0 & 0 & 0 \\
g_{13} & g_{23} & g_{33} & 0 & 0 & 0 \\
0 & 0 & 0 & g_{4} & 0 & 0 \\
0 & 0 & 0 & 0 & g_{5} & 0 \\
0 & 0 & 0 & 0 & 0 & g_{6}
\end{array}\right)
$$

Taking into account that the antiferromagnetic vector in $\mathrm{TmFeO}_{3}$ lies in the $x-z$ plane, such that $\mathbf{G}=(\cos \theta, 0, \sin \theta)$, we obtain

$$
\begin{aligned}
W_{\text {int }}= & \cos ^{2} \theta\left(g_{11} E_{x}^{2}+g_{12} E_{y}^{2}+g_{13} E_{z}^{2}\right) \\
& +\sin ^{2} \theta\left(g_{12} E_{x}^{2}+g_{22} E_{y}^{2}+g_{33} E_{z}^{2}\right)+\frac{1}{2} g_{5} \sin 2 \theta E_{x} E_{z}
\end{aligned}
$$

which can be rearranged as

$$
W_{\mathrm{int}}=f(\mathbf{E})+\sin ^{2} \theta\left(\chi_{x} E_{x}^{2}+\chi_{y} E_{y}^{2}+\chi_{z} E_{z}^{2}\right)+\frac{1}{2} g_{5} \sin 2 \theta E_{x} E_{z}
$$

where $\chi_{x}=g_{12}=-g_{11}, \chi_{y}=g_{22}-g_{11}, \chi_{z}=g_{33}-g_{13}$. The function

$f(\mathbf{E})=\left(g_{11} E_{x}^{2}+g_{12} E_{y}^{2}+g_{13} E_{z}^{2}\right)$ does not depend on $\theta$ and can be omitted.

Importantly, the interaction term in equation (10) can be present in any crystal with $D_{2 h}^{16}$ point group, regardless of the exact microscopic origin (electronic excitations, phonons, excitons and so on) of coupling between the antiferromagnetic vector and the electric field. In our experiment the terahertz magnetic field is linearly polarized along the $x$ axis and therefore $E_{x}=0$. Thus, the interaction energy (10) reduces to

$$
W_{\text {int }}=\left(\chi_{y} E_{y}^{2}+\chi_{z} E_{z}^{2}\right) \sin ^{2} \theta
$$

and can be seen as a modulation of the anisotropy energy $W_{\mathrm{A}}(\theta)=K_{1} \sin ^{2} \theta+K_{2} \sin ^{4} \theta$. The terahertz electric field changes the anisotropy parameter $K_{1}$ in equation (3) by $\Delta K_{1}=\chi_{y} E_{y}^{2}+\chi_{z} E_{z}^{2}$.

The coupling described by equation (11) generates a torque acting on spins, which results in an additional term on the right-hand side of the generalized sineGordon equation (7):

$$
\ddot{\theta}+\omega_{\mathrm{E}} \alpha \dot{\theta}+\omega_{\mathrm{E}} \omega_{\mathrm{A}} w(\theta, T)=-\omega_{\mathrm{E}} \omega_{\mathrm{A}} \sin \theta \cos \theta a(t)
$$

with

$$
a(t)=1 / K_{2}\left(\chi_{y} E_{y}^{2}(t)+\chi_{z} E_{z}^{2}(t)\right)
$$

being proportional to the terahertz intensity time trace. In equation (12) we do not include the additive Zeeman torque considered above to isolate the effect of the terahertz-induced transient anisotropy. If the duration of the terahertz pulse and the relaxation time of the excited $\mathrm{Tm}^{3+}$ states are much shorter than the period of the quasi-ferromagnetic mode, one can show that the generated torque acts as an instantaneous excitation described by the initial conditions

$$
\theta(t=0)=\theta_{0}, \dot{\theta}(t=0)=-\omega_{\mathrm{E}} \omega_{\mathrm{A}} \sin \theta_{0} \cos \theta_{0} \int_{0}^{\tau_{\mathrm{THz}}} a(t) \mathrm{d} t
$$

where $\tau_{\mathrm{THz}}$ is the duration of the terahertz pulse. The transient anisotropy torque depends on the temperature as $\sin \theta_{0}(T) \cos \theta_{0}(T)$, which is shown in Fig. $3 \mathrm{~d}$. It is non-zero only in the temperature interval corresponding to the intermediate magnetic phase $\Gamma_{24}$. Obviously, the torque is quadratic with respect to the terahertz field $\mathbf{E}$ (equations (13) and (14)). The Zeeman torque and the transient anisotropy torque are additive, but scale differently with the terahertz peak field.

Finally we note that our phenomenological theory correctly reproduces the spin dynamics on timescale longer than the duration of the terahertz-driven perturbation of the anisotropy fields. To describe the strongly non-equilibrium state during the interaction of the medium with the intense terahertz pulse, one 
may need to account for the terahertz carrier wave within a time-dependent manybody theory.

Estimation of the strength of the Zeeman torque. Applying a terahertz low-pass filter with a nominal cutoff frequency of $0.3 \mathrm{THz}$ allows us to selectively excite the q-FM mode (Fig. 4a, inset). In this measurement, the q-FM amplitude amounts to $A_{z}=8 \%$ of the one induced by the unfiltered terahertz transients (Fig. 4a). Taking into account the filter transmission of $T_{\mathrm{F}} \approx 75 \%$ at the $\mathrm{q}-\mathrm{FM}$ resonance frequency, we conclude that the anisotropy-induced torque is $\left(1-A_{\mathrm{z}} / T_{\mathrm{F}}\right) /\left(A_{\mathrm{z}} / T_{\mathrm{F}}\right) \approx 8$ times as large as the Zeeman interaction for $\mathrm{THz}$ pulses featuring peak magnetic fields of $0.3 \mathrm{~T}$.

\section{References}

31. Zvezdin, A. K. Dynamics of domain walls in weak ferromagnets. JETP Lett. 29, 553-556 (1979).

32. Andreev, A. F. \& Marchenko, V. I. Symmetry and the macroscopic dynamics of magnetic materials. Sov. Phys. Usp. 23, 21-31 (1980). 